\title{
Relativistic Motion with Viscosity. I Newton's Law of Resistance
}

\author{
Lorenzo Zaninetti \\ Physics Department, Turin, Italy \\ Email:1.zaninetti@alice.it
}

How to cite this paper: Zaninetti, L. (2021) Relativistic Motion with Viscosity. I Newton's Law of Resistance. International Journal of Astronomy and Astrophysics, $11,435-444$.

https://doi.org/10.4236/ijaa.2021.113021

Received: July 31, 2021

Accepted: September 21, 2021

Published: September 24, 2021

Copyright (อ 2021 by author(s) and Scientific Research Publishing Inc. This work is licensed under the Creative Commons Attribution International License (CC BY 4.0).

http://creativecommons.org/licenses/by/4.0/

\section{(c) (i) Open Access}

\begin{abstract}
The equation of motion for a relativistic neutral particle that moves in a medium characterized by a friction proportional to the square of the velocity is analyzed. The relativistic trajectory is derived in a numerical way and in the form of a Taylor series. The astrophysical applications cover the trajectory of SN 1993J and the light curve of gamma ray bursts.
\end{abstract}

\section{Keywords}

Supernovae, General, Supernovae, (Individual: SN1993j), Gamma-Ray Burst, (Individual: GRB 130427A), Gamma-Ray Burst, (Individual: GRB 060729)

\section{Introduction}

Relativistic viscosity has the following applications: change in the mean particle momentum and spreading around the mean for the cosmic rays (CR) [1]; acceleration of CR in shear flows, such as active galactic nuclei (AGN), gamma ray burst (GRB) and jets [2] [3]; interaction of a neutral particle with the microwave background radiation (CMB) [4] [5]; and generation of the CMB in the expanding universe [6]. The Lagrangian and a Hamiltonian for a relativistic particle moving in a dissipative medium characterized by a force that depends on the square of the velocity of the particle have been derived [7]. This paper is a highly idealized attempt to model SN light curves by assuming that the resistivity of the ambient interstellar medium is quadratic to the velocity of the SN envelope. Section 2 derives a relativistic equation of motion in the presence of viscosity proportional to the square of the velocity. Section 3 applies the relativistic results to the motion of SN 1993J, to the light curve of GRB 130427A and GRB 060729. The CMB is not related with the model that is presented here. 


\section{The Equation of Motion}

We assume a one dimensional motion with a resistive force of Newtonian type, $F_{\text {res }}=-B m_{0} v(t)^{2}$, where $B$ is a constant, $m_{0}$ is the considered mass and $v(t)$ is the velocity. In the following, we will consider only positive and decreasing velocities. Newton's second law in special relativity is:

$$
F=\frac{\mathrm{d} p}{\mathrm{~d} t}=\frac{\mathrm{d}}{\mathrm{d} t}(m v(t))=\frac{\mathrm{d}}{\mathrm{d} t}\left(\frac{m_{0} v(t)}{\sqrt{1-\frac{v(t)^{2}}{c^{2}}}}\right)=\frac{\mathrm{d}}{\mathrm{d} t}\left(m_{0} v \gamma\right),
$$

where $F$ is the force, $p$ is the relativistic momentum, $m$ is the relativistic mass, $m_{0}$ is the rest mass, $c$ is the velocity of light, $v(t)$ is the velocity and $\gamma=\frac{1}{\sqrt{1-\frac{v(t)^{2}}{c^{2}}}}$ is the Lorentz factor; see equation (7.16) in [8]. The first order differential equation in the velocity that governs the motion is

$$
\frac{\frac{\mathrm{d}}{\mathrm{d} t} v(t)}{\left(1-\frac{(v(t))^{2}}{c^{2}}\right)^{\frac{3}{2}}}=-B(v(t))^{2}
$$

The solution to this first order differential equation for $v(t)$ in an implicit form is

$$
\frac{c^{2}-2 v^{2}}{c v} \frac{1}{\sqrt{c^{2}-v^{2}}}-\frac{c^{2}-2 v_{0}^{2}}{c v_{0}} \frac{1}{\sqrt{c^{2}-v_{0}^{2}}}=B\left(t-t_{0}\right),
$$

where $v_{0}$ is the velocity at $t=t_{0}$. An explicit solution for the velocity can be obtained by considering the physical solution of the previous algebraic equation of fourth degree in $V$

$$
v^{4}+p v^{2}+q=0
$$

with

$$
p=-c^{2}
$$

and

$$
q=\frac{\left(c^{2}-v_{0}^{2}\right)^{\frac{3}{2}} c^{3} v_{0}^{2}}{N Q}
$$

where

$$
\begin{aligned}
N Q= & B^{2} \sqrt{c^{2}-v_{0}^{2}} c^{3} t^{2} v_{0}^{2}-2 B^{2} \sqrt{c^{2}-v_{0}^{2}} c^{3} t t_{0} v_{0}^{2}+B^{2} \sqrt{c^{2}-v_{0}^{2}} c^{3} t_{0}^{2} v_{0}^{2} \\
& -B^{2} \sqrt{c^{2}-v_{0}^{2}} c t^{2} v_{0}^{4}+2 B^{2} \sqrt{c^{2}-v_{0}^{2}} c t t_{0} v_{0}^{4}-B^{2} \sqrt{c^{2}-v_{0}^{2}} c t_{0}^{2} v_{0}^{4} \\
& +2 B c^{4} t v_{0}-2 B c^{4} t_{0} v_{0}-6 B c^{2} t v_{0}^{3}+6 B c^{2} t_{0} v_{0}^{3}+4 B t v_{0}^{5} \\
& -4 B t_{0} v_{0}^{5}+\sqrt{c^{2}-v_{0}^{2}} c^{3} .
\end{aligned}
$$


Before we continue, we will introduce the following simplification.

Conjecture 1. In the presence of more than one solution for the temporal evolution of the velocity/space, we select the physical one that has a positive decreasing/increasing behavior. According to this statement, the physical solution is

$$
v(t)=\frac{1}{2} \sqrt{-2 \sqrt{p^{2}-4 q}-2 p} .
$$

We already know that $v=v_{0}$ at $t=t_{0}$. Once we know that $v=v_{1}$ at $t=t_{1}$, it is possible to derive the unknown parameter $B$ from the previous formula

$$
B=\frac{c^{4} v_{1}-2 c^{2} v_{0}^{2} v_{1}-c^{2} v_{1}^{3}+2 v_{0}^{2} v_{1}^{3}-\sqrt{c^{2}-v_{0}^{2}} \sqrt{v_{0}^{2}\left(c^{2}-v_{1}^{2}\right)\left(c^{2}-2 v_{1}^{2}\right)^{2}}}{\sqrt{c^{2}-v_{0}^{2}} v_{0} v_{1}\left(t_{0}-t_{1}\right)\left(c^{2}-v_{1}^{2}\right) c} .
$$

At this moment, we are unable to obtain an analytical solution for $r(t)$. Therefore, the trajectory is obtained by a numerical integration of Equation (8).

An approximation for the trajectory is represented by a Taylor series expansion about $r=r_{0}$ of order 2

$$
\begin{aligned}
& r\left(t ; t_{0}, r_{0}, v_{0}, B\right)=r_{0}+v_{0}\left(t-t_{0}\right)-\frac{B v_{0}^{2}\left(\frac{c^{2}-v_{0}^{2}}{c^{2}}\right)^{\frac{3}{2}}\left(t-t_{0}\right)^{2}}{2} \\
& +\frac{B^{2} v_{0}^{3}\left(c^{2}-v_{0}^{2}\right)^{2}\left(2 c^{2}-5 v_{0}^{2}\right)\left(t-t_{0}\right)^{3}}{6 c^{6}},
\end{aligned}
$$

which means the following approximate velocity as function of time

$$
\begin{aligned}
v\left(t ; t_{0}, r_{0}, v_{0}, B\right)= & v_{0}-B v_{0}^{2}\left(\frac{c^{2}-v_{0}^{2}}{c^{2}}\right)^{\frac{3}{2}}\left(t-t_{0}\right) \\
& +\frac{B^{2} v_{0}^{3}\left(c^{2}-v_{0}^{2}\right)^{2}\left(2 c^{2}-5 v_{0}^{2}\right)\left(t-t_{0}\right)^{2}}{2 c^{6}} .
\end{aligned}
$$

We now analyze the mildly relativistic case for which the relativistic momentum in the case of a unit mass is

$$
p(t)=v(t)+\frac{v(t)^{3}}{2 c^{2}},
$$

which means the following first order differential equation for the velocity

$$
\frac{\mathrm{d}}{\mathrm{d} t} v(t)+\frac{3 v(t)^{2}\left(\frac{\mathrm{d}}{\mathrm{d} t} v(t)\right)}{2 c^{2}}=-B v(t)^{2} .
$$

The velocity in the mildly relativistic case is

$$
v(t)=\frac{2 B c^{2} t_{0} v_{0}-2 B c^{2} v_{0} t-2 c^{2}+3 v_{0}^{2}+\sqrt{V A}}{6 v_{0}}
$$

where 


$$
\begin{aligned}
V A= & 4 B^{2} c^{4} t^{2} v_{0}^{2}-8 B^{2} c^{4} t t_{0} v_{0}^{2}+4 B^{2} c^{4} t_{0}^{2} v_{0}^{2}+8 B c^{4} t v_{0}-8 B c^{4} t_{0} v_{0} \\
& -12 B c^{2} t v_{0}^{3}+12 B c^{2} t_{0} v_{0}^{3}+4 c^{4}+12 c^{2} v_{0}^{2}+9 v_{0}^{4} .
\end{aligned}
$$

The indefinite integral of the velocity is

$$
\begin{aligned}
I(t)= & \int v(t) \mathrm{d} t \\
= & \frac{1}{24 B v_{0}^{2} c^{2}}\left(-4 B^{2} c^{4} t^{2} v_{0}^{2}+8 B^{2} c^{4} t t_{0} v_{0}^{2}-8 B c^{4} t v_{0}+12 B c^{2} t v_{0}^{3}\right. \\
& +2 B \sqrt{I A} c^{2} t v_{0}-2 B \sqrt{I A} c^{2} t_{0} v_{0}+24 \ln \left(\sqrt{I A}-3 v_{0}^{2}\right. \\
& \left.\left.+\left(2 t-2 t_{0}\right) B c^{2} v_{0}+2 c^{2}\right) v_{0}^{2} c^{2}+2 \sqrt{I A} c^{2}-3 \sqrt{I A} v_{0}^{2}\right),
\end{aligned}
$$

where

$$
I A=4\left(1+B v_{0}\left(t-t_{0}\right)\right)^{2} c^{4}-12 v_{0}^{2}\left(-1+B v_{0}\left(t-t_{0}\right)\right) c^{2}+9 v_{0}^{4},
$$

which leads to the following trajectory in the mildly relativistic case

$$
r(t)=I(t)-I\left(t_{0}\right) .
$$

The unknown parameter $B$ in the mildly relativistic case is

$$
B=\frac{-v_{0}\left(2 c^{2} v_{0}-2 c^{2} v_{1}+3 v_{0}^{2} v_{1}-3 v_{0} v_{1}^{2}\right)}{2 c^{2} v_{0}^{2} v_{1}\left(t_{0}-t_{1}\right)} .
$$

\section{The Relativistic Luminosity}

The relativistic transfer of energy through a surface, $A$, is

$$
L_{m, r}=A \gamma^{2}\left(\rho c^{2}+p\right) v,
$$

where $p$ is the pressure. For sake of simplicity, we take $p=0$, and $\gamma$ is the Lorentz factor, see eqn. A31 in [9] or eqn. (43.44) in [10]. In the case of a spherical cold expansion

$$
L_{m, r}=4 \pi r(t)^{2} \frac{1}{1-\beta(t)^{2}} \rho(t) c^{3} \beta(t) .
$$

We now assume the following power-law behavior for the density in the advancing layer of radius $r$

$$
\rho(r)=\rho_{0}\left(\frac{r_{0}}{r}\right)^{d}
$$

which has the following temporal scaling

$$
\rho(t)=\rho_{0}\left(\frac{r_{0}}{r\left(t ; t_{0}, r_{0}, v_{0}, B\right)}\right)^{d},
$$

where $r(t)$ is given by the Taylor series represented by Equation (10). The mechanical relativistic luminosity is

$$
L_{m, r}=4 \pi r(t)^{2} \frac{1}{1-\beta(t)^{2}} \rho_{0}\left(\frac{r_{0}}{r}\right)^{d} c^{3} \beta(t) .
$$

Once we insert formulae (10) and (11) in this equation, we obtain an ap- 
proximate expression for the mechanical relativistic energy, which has a complicated expression that is not here reported. We now parameterize the presence of the absorption introducing a the optical thickness $\tau_{v}$. The observed luminosity is assumed to be

$$
L_{o b s}=C_{o b s} L_{m, r}\left(1-\mathrm{e}^{-\tau_{v}}\right) \text {, }
$$

where $C_{o b s}$, which is here assumed to be constant in the interval of time considered here, allows the match between theory and observations. The optical thickness $\tau_{v}$ takes the value $\infty$ in the case of an optically thin medium or can be function of time to simulate the complex behavior of the observed luminosity; more details can be found in [11]. The observed absolute magnitude is

$$
M_{o b s}=-\log _{10}\left(L_{o b s}\right)+k_{o b s},
$$

where $k_{o b s}$ is a constant of match between theory and observations.

\section{Astrophysical Applications}

The chosen astrophysical units are pc for length and yr for time. With these units, the initial velocity is $v_{0}\left(\mathrm{~km} \cdot \mathrm{s}^{-1}\right)=9.7968 \times 10^{5} v_{0}\left(\mathrm{pc} \cdot \mathrm{yr}^{-1}\right)$.

As a first example, we apply the above results to the deduction of the parameter $B$ to SN 1993J for which observational times and velocities are available [12] [13], see Table 1. A test for the quality of the fits is represented by the merit function $\chi^{2}$

$$
\chi^{2}=\sum_{j} \frac{\left(r_{t h}-r_{o b s}\right)^{2}}{\sigma_{o b s}^{2}},
$$

where $r_{t h}, r_{o b s}$ and $\sigma_{o b s}$ are the theoretical radius, the observed radius and the observed uncertainty, respectively. Once $B$ is derived on an observational basis, Figure 1 reports the analytical velocity and Figure 2 reports the numerical trajectory, with data as given in Table 1.

A comparison between the numerical solution with the Runge Kutta method and a Taylor series is reported in Figure 3.

The second example is applied to the light curve (LC) of GRB 130427A, which was the most luminous gamma-ray burst in the last 30 years, see Figure 1 in [14]. Figure 4 reports the $\mathrm{X}$-flux as a function of time and the relative theoretical

Table 1. Numerical values of the parameters for the fit and the theoretical model applied to SN 1993J.

\begin{tabular}{ccc}
\hline model & values & $\chi^{2}$ \\
\hline Fit by a power law & $\alpha_{\text {fit }}=0.828 ; C=0.015$ & 43 \\
Newtonian viscosity & $r_{0}=3.0 \times 10^{-3} \mathrm{pc} ; v_{0}=13800 \mathrm{~km} / \mathrm{s}$ & 45.59 \\
B evaluation & $v_{0}=2.39 \times 10^{-2} \mathrm{pc} / \mathrm{yr}, v_{1}=8.6 \times 10^{-3} \mathrm{pc} / \mathrm{yr}$ & \\
B evaluation & $t_{0}=2.6 \times 10^{-2} \mathrm{yr}, t_{1}=10.58 \times 10^{-2} \mathrm{yr}$ & $B=7.082 \mathrm{pc}^{-1}$ \\
\hline
\end{tabular}




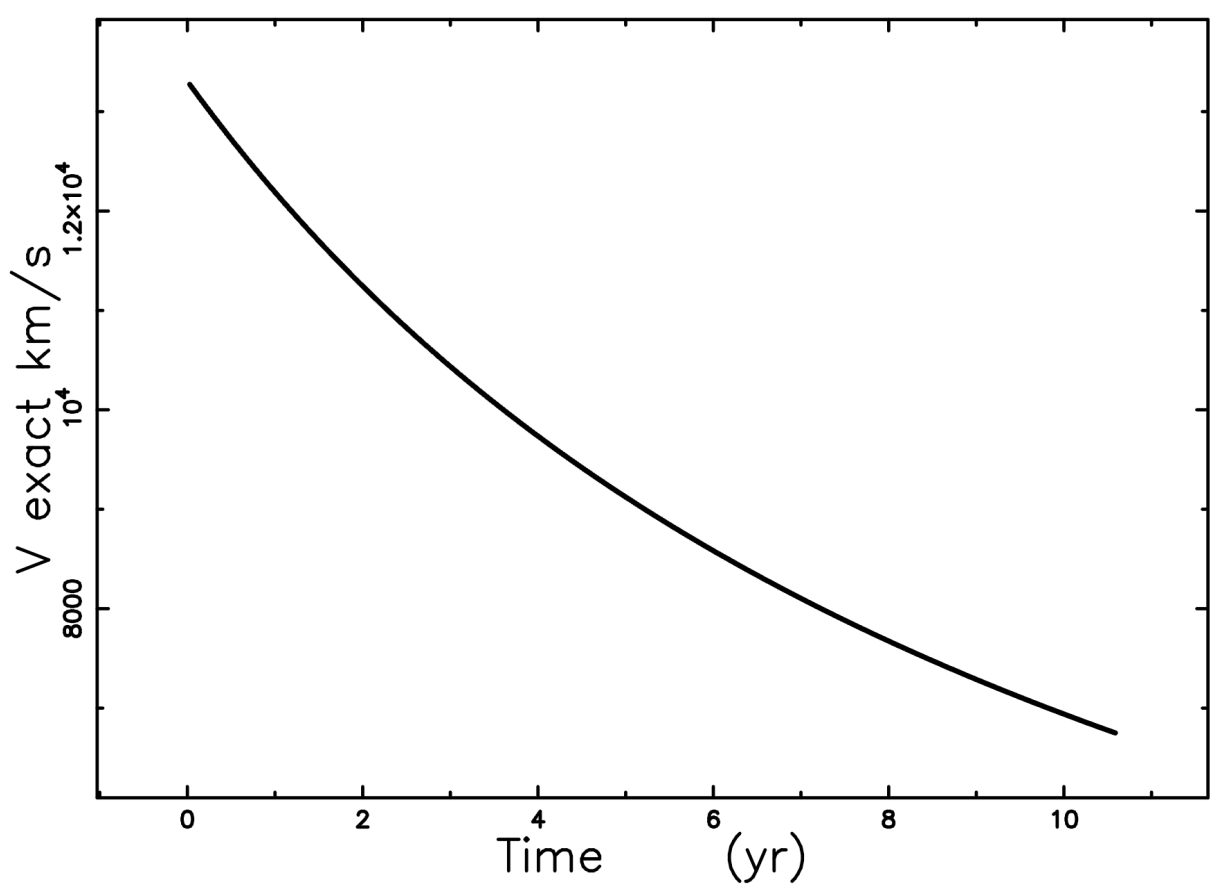

Figure 1. Analytical velocity versus time (full line) for SN 1993J as given by formula (8).

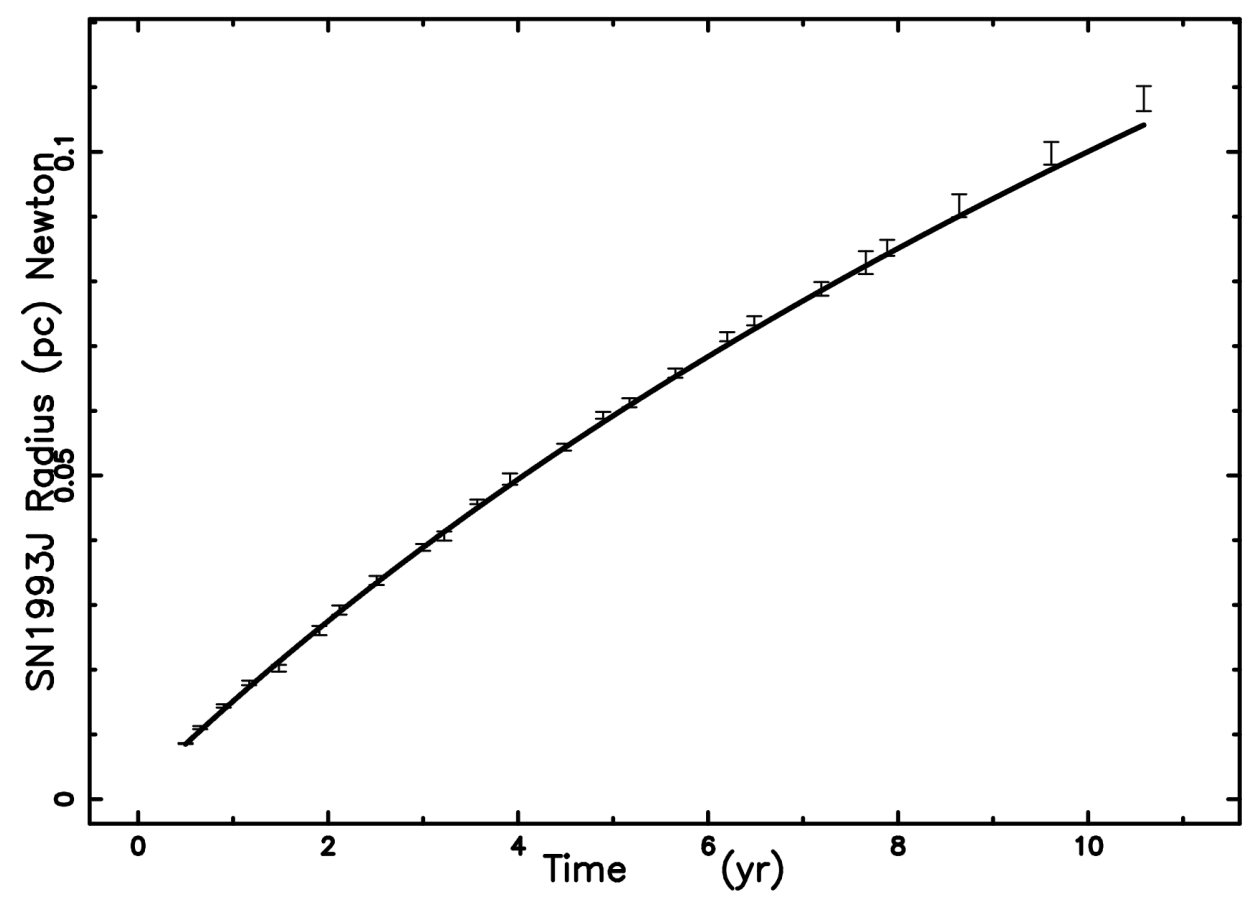

Figure 2. Numerical radius (full line) and astronomical data of SN 1993J with vertical error bars.

data with data as in Table 2.

The third example is dedicated to the LC for GRB 060729 as observed by the Ultraviolet and Optical Telescope (UVOT) in the time interval [10-2 26$]$ days, see Figure 1 in [15]. Figure 5 presents the LC of UVOT (U) apparent magnitude for GRB 060729 with data as in Table 3, and Figure 6 presents the temporal 


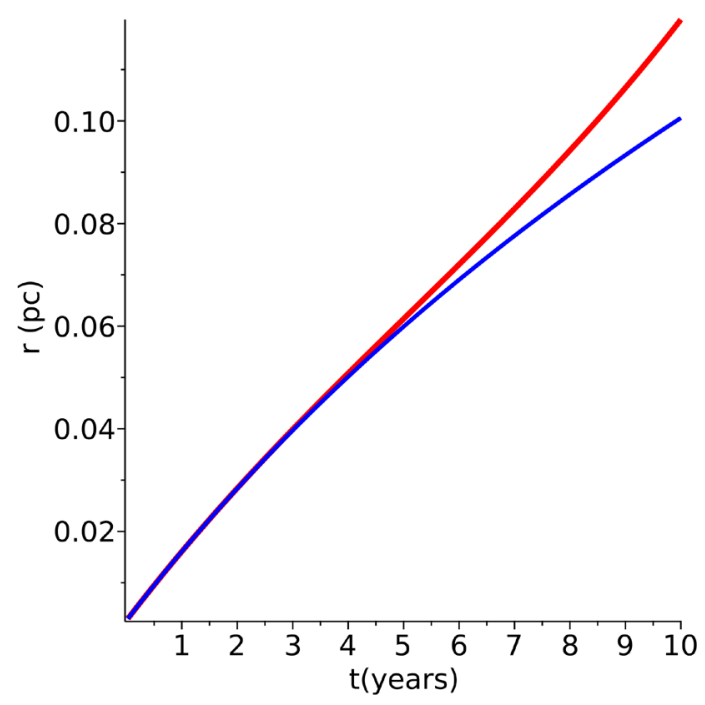

Figure 3. Numerical radius (blue line) and Taylor solution (red line) as represented by Equation (10).

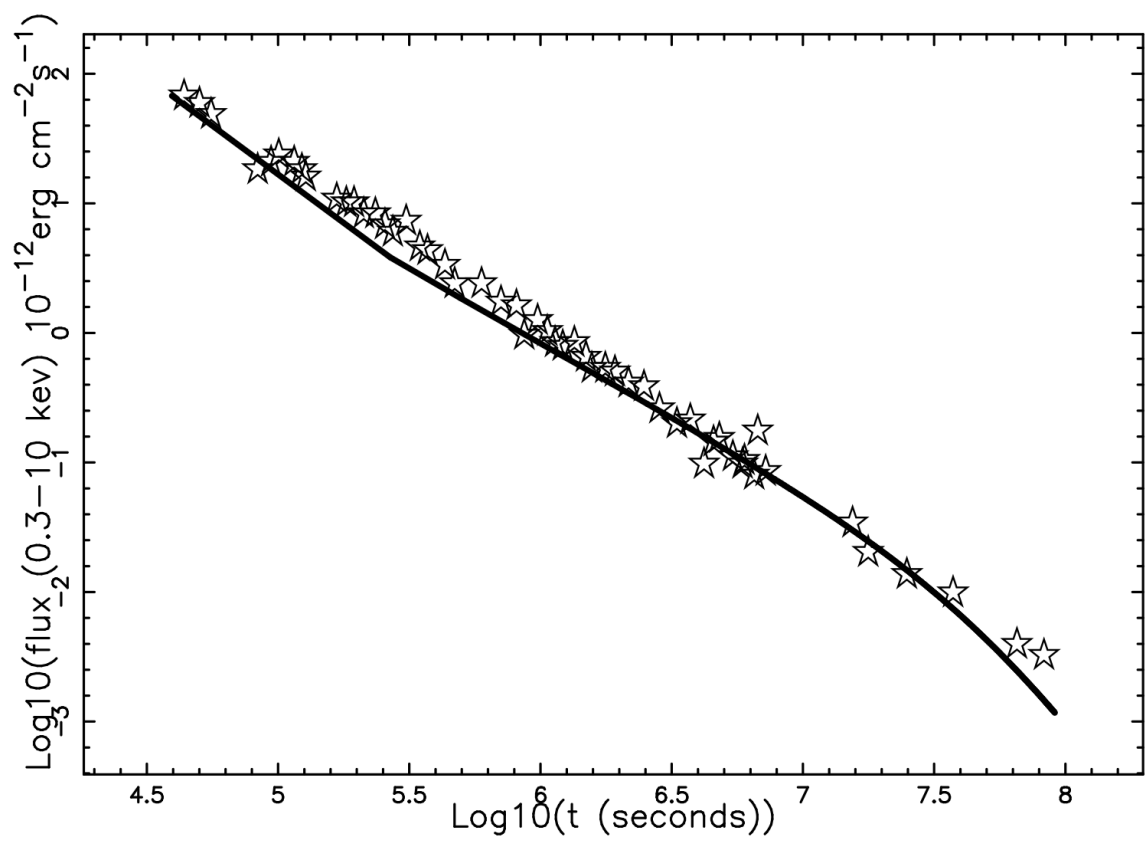

Figure 4. Flux in the X-ray as function of time in seconds for GRB 130427A (empty stars) and theoretical curve as given by Equation (25) (full line) when $\tau_{v}=\infty$ with data as in Table 2.

Table 2. Numerical values of the parameters for the theoretical model applied to GRB 130427A.

$$
r_{0}=1.0 \times 10^{-4} \mathrm{pc} ; t_{0}=1.0 \times 10^{-4} \mathrm{~s} ; \beta_{0}=0.99 ; B=7.082 \mathrm{pc}^{-1} ; d=3.1
$$

Table 3. Numerical values of the parameters for the theoretical model applied to GRB 060729.

$$
r_{0}=1.0 \times 10^{-4} \mathrm{pc} ; t_{0}=2.0 \times 10^{-6} \mathrm{~s} ; \beta_{0}=0.99 ; B=7.082 \mathrm{pc}^{-1} ; d=3
$$




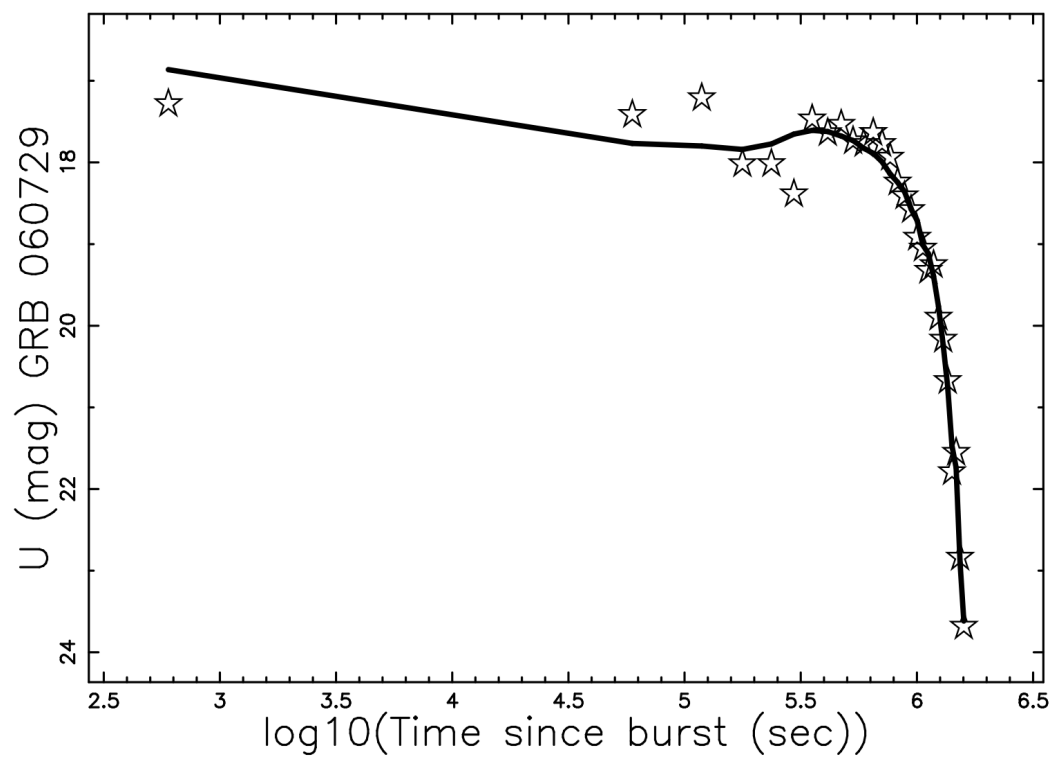

Figure 5. The LC of UVOT (U) + HST (F330W) for GRB 060729 (empty stars) and theoretical curve with radius as given by the relativistic numerical model with data as in Table 3. The theoretical magnitude is given by Equation (26) (full line).

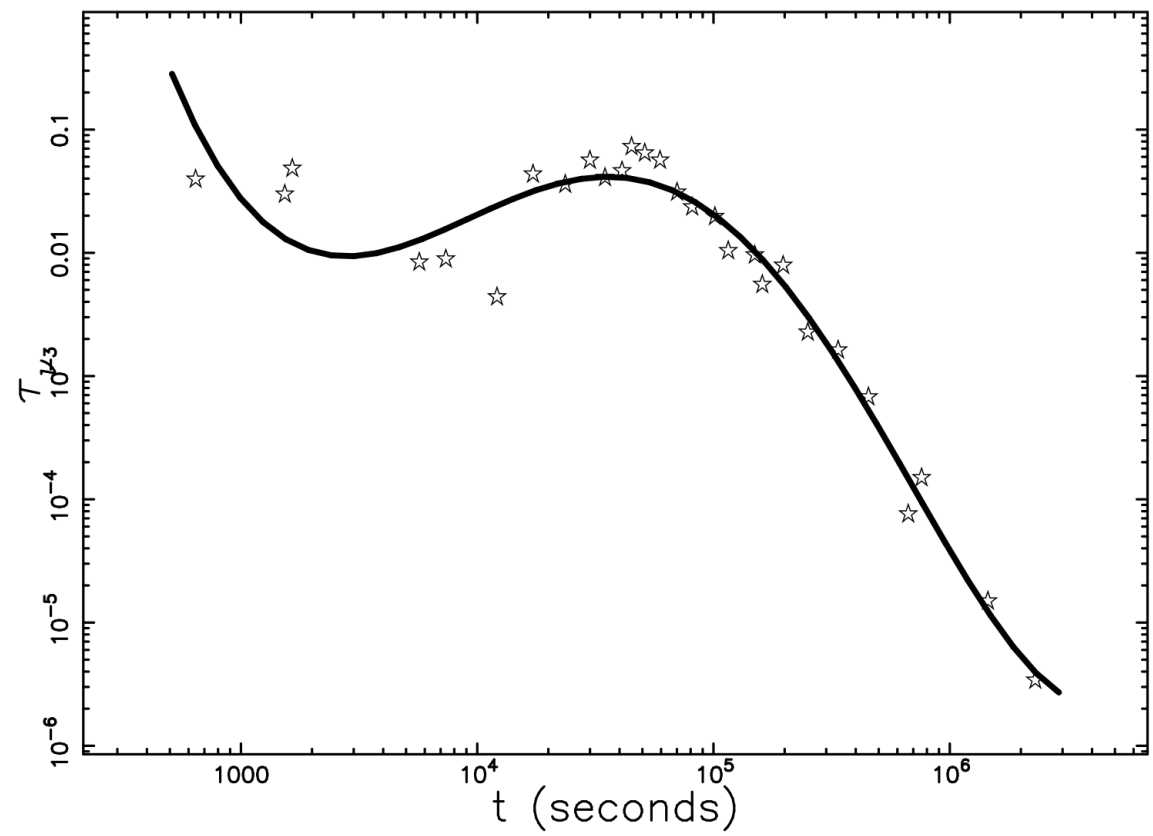

Figure 6. The time dependence of $\tau_{v}$ (empty stars) for GRB 060729 and a logarithmic polynomial approximation of degree 5 (full line). Parameters as in Table 3.

behavior of the optical depth.

\section{Conclusions}

Physics. We have derived velocity as a function of time for a relativistic neutral particle that moves in a dissipative medium in the presence of friction, which depends on the square velocity of the particle. The trajectory (i.e., space as a 
function of time) can be deduced by numerical integration of the velocity or by a Taylor series of the differential equation of the second order.

Astrophysics. An application of the obtained results to SN 1993J allows us to derive the constant $B$ and draw a comparison between the observed and theoretical trajectory, see Table 1 and Figure 2. The LCs of GRB 130427A and GRB 060729 were simulated, see Figure 4 and Figure 5.

\section{Conflicts of Interest}

The author declares no conflicts of interest regarding the publication of this paper.

\section{References}

[1] Earl, J.A., Jokipii, J.R. and Morfill, G. (1988) Cosmic-Ray Viscosity. The Astrophysical Journal Letters, 331, L91-L94. https://doi.org/10.1086/185242

[2] Rieger, F. and Duffy, P. (2007) Cosmic-Ray Acceleration and Viscosity. In: Aschenbach, B., Burwitz, V., Hasinger, G. and Leibundgut, B., Eds., Relativistic Astrophysics Legacy and Cosmology_Einstein's, Springer, Berlin, 477-479.

https://doi.org/10.1007/978-3-540-74713-0 110

[3] Webb, G.M., Barghouty, A.F., Hu, Q. and le Roux, J.A. (2018) Particle Acceleration Due to Cosmic-Ray Viscosity and Fluid Shear in Astrophysical Jets. The Astrophysical Journal, 855, 31. https://doi.org/10.3847/1538-4357/aaae6c

[4] Dedkov, G. and Kyasov, A. (2010) Tangential Force and Heating Rate of a Neutral Relativistic Particle Mediated by Equilibrium Background Radiation. Nuclear Instruments and Methods in Physics Research Section B: Beam Interactions with Materials and Atoms, 268, 599. https://doi.org/10.1016/j.nimb.2009.12.011

[5] Volokitin, A.I. (2015) Blackbody Friction Force on a Relativistic Small Neutral Particle. Physical Review A, 91, Article ID: 032505. https://doi.org/10.1103/PhysRevA.91.032505

[6] Kolekar, S., Shankaranarayanan, S. and Chitre, S.M. (2020) Generation of CMB and Cosmological Constant via Bulk Viscosity. General Relativity and Gravitation, 52, 98. https://doi.org/10.1007/s10714-020-02749-0

[7] López, G.V., Montes, G.C. and Zanudo, J.G.T. (2015) One Dimensional Relativistic Free Particle in a Quadratic Dissipative Medium. Journal of Modern Physics, 6, 121-125. https://doi.org/10.4236/jmp.2015.62016

[8] French, A.P. (1968) Special Relativity. CRC, New York.

[9] De Young, D.S. (2002) The Physics of Extragalactic Radio Sources. University of Chicago Press, Chicago.

[10] Mihalas, D. and Mihalas, B. (2013) Foundations of Radiation Hydrodynamics. Dover Books on Physics, Dover Publications, New York.

[11] Zaninetti, L. (2021) Energy Conservation in the Thin Layer Approximation: IV. The Light Curve for Supernovae. International Journal of Astronomy and Astrophysics, 11, 37. https://doi.org/10.4236/ijaa.2021.111003

[12] Marcaide, J.M., Mart-Vidal, I., Alberdi, A. and Pérez-Torres, M.A. (2009) A Decade of SN 1993J: Discovery of Radio Wavelength Effects in the Expansion Rate. $A \& A$, 505, 927. https://doi.org/10.1051/0004-6361/200912133

[13] Mart-Vidal, I., Marcaide, J.M., Alberdi, A., Guirado, J.C., Pérez-Torres, M.A. and 
Ros, E. (2011) Radio Emission of SN1993J: The Complete Picture. II. Simultaneous Fit of Expansion and Radio Light Curves $A \& A, \mathbf{5 2 6}$, A143. https://doi.org/10.1051/0004-6361/201014517

[14] De Pasquale, M., Page, M., Kann, D., Oates, S., Schulze, S., Zhang, B., Cano, Z., Gendre, B., Malesani, D., Rossi, A., Gehrels, N., Troja, E., Piro, L., Boër, M. and Stratta, G. (2017) Challenging the Forward Shock Model with the 80 Ms Follow up of the X-Ray Afterglow of Gamma-Ray Burst 130427A. Galaxies, 5, 6. https://doi.org/10.3390/galaxies5010006

[15] Cano, Z., Bersier, D., Guidorzi, C., et al. (2011) A Tale of Two GRB-SNe at a Common Redshift of $\mathrm{z}=0.54$. MNRAS, 413, 669 . 